AUTHOR CORRECTION

\title{
Correction to: Randomized phase 2 trial of pevonedistat plus azacitidine versus azacitidine for higher-risk MDS/CMML or low-blast AML
}

Mikkael A. Sekeres (D), Justin Watts, Atanas Radinoff, Montserrat Arnan Sangerman, Marco Cerrano (D), Patricia Font Lopez (D), Joshua F. Zeidner (D), Maria Diez Campelo (D), Carlos Graux, Jane Liesveld, Dominik Selleslag, Nikolay Tzvetkov, Robert J. Fram, Dan Zhao, Jill Bell, Sharon Friedlander, Douglas V. Faller (iD) and Lionel Adès

(c) The Author(s), under exclusive licence to Springer Nature Limited 2021

Leukemia (2021) 35:3637; https://doi.org/10.1038/s41375-021-01473-1

Correction to: Leukemia https://doi.org/10.1038/s41375-021-01125-4, published online 22 January 2021

In Supplementary Table 2, the $n$ numbers for the response-evaluable patients with higher-risk MDS were swapped for the pevonedistat + azacitidine arm and the azacitidine alone arm. Instead of $n=30$ and $n=29$ as originally published, these have now been corrected to $n=29$ for pevonedistat + azacitidine and $n=30$ for azacitidine. No changes were required to the response rate percentages as these were calculated using the correct denominators.
The updated files are attached to this correction.

\section{ADDITIONAL INFORMATION}

Supplementary information The online version contains supplementary materia available at https://doi.org/10.1038/s41375-021-01473-1. 